\title{
Financial integration and the ASEAN-5 equity markets
}

\begin{abstract}
The existence of long-run relationships among the ASEAN-5 equity markets is empirically investigated. This study utilized weekly data spanning January 1988 to August 1999. The results of Granger noncausality test due to Toda and Yamamoto (Journal of Econometrics, $66,225-50,1995)$ reveal that the Singapore equity market was not affected by other markets except by the Philippines in the long run. This result shows that there exist opportunities for beneficial international portfolio diversification within the context of the Asean-5 equity markets.
\end{abstract}

Keyword: ASEAN; capital market; economic integration; stock market 